\title{
Star Formation in Nearby Early-Type Galaxies: Mapping in UV, Optical and CO
}

M. Bureau ${ }^{1}$, R. Bacon ${ }^{2}$, M. Cappellari ${ }^{3}$, F. Combes $^{4}$, R. L. Davies ${ }^{1}$, P. T. de Zeeuw ${ }^{3}$, E. Emsellem ${ }^{2}$, J. Falcón-Barroso ${ }^{5}$, H. Jeong ${ }^{6}$, D. Krajnović ${ }^{1}$, H. Kuntschner ${ }^{7}$, R. M. McDermid ${ }^{3}$, R. F. Peletier ${ }^{8}$, M. $\operatorname{Sarzi}^{9}$, K. L. Shapiro ${ }^{10}$, G. van de Ven ${ }^{11}$, S. K. Yi ${ }^{6}$, and L. M. Young ${ }^{12}$

${ }^{1}$ Sub-Department of Astrophysics, University of Oxford, United Kingdom

${ }^{2}$ Centre de Recherche Astronomique de Lyon - Observatoire, France

${ }^{3}$ Sterrewacht Leiden, Universiteit Leiden, The Netherlands

${ }^{4}$ Observatoire de Paris, France

${ }^{5}$ European Space Research and Technology Centre, The Netherlands

${ }^{6}$ Department of Astronomy, Yonsei University, South Korea

${ }^{7}$ ST-ECF, European Southern Observatory, Germany

${ }^{8}$ Kapteyn Astronomical Institute, Universiteit Groningen, The Netherlands

${ }^{9}$ Centre for Astrophysics Research, University of Hertfordshire, United Kingdom

${ }^{10}$ Department of Astronomy, University of California Berkeley, U.S.A.

${ }^{11}$ Department of Astrophysical Sciences, Princeton University, U.S.A.

${ }^{12}$ Department of Physics, New Mexico Institute of Mining and Technology, U.S.A.

\begin{abstract}
The SAURON integral-field survey reveals that small $\left(\sim 0.1 R_{\mathrm{e}}\right)$ kinematically decoupled cores (KDCs) in early-type galaxies are increasingly young toward the center and are typically found in fast-rotating galaxies, while large KDCs $\left(\sim 0.5 R_{\mathrm{e}}\right)$ have homogeneously old stars and are present in non-rotating galaxies (McDermid et al. 2006). GALEX UV imaging further allows the direct identification of regions of recent star formation ( $\leqslant 0.5 \mathrm{Gyr}$ ). In NGC 2974 for example, young stars are identified in the center and an outer ring (Jeong et al. 2006). Nuclear and inner ionised-gas rings (Sarzi et al. 2006) then suggest that current star formation is bar-driven. The CO detection rate of SAURON early-type galaxies is $\approx 40 \%$ (Combes et al. in prep.). Synthesis imaging reveals that it is generally contained in a well-ordered central disk, both in galaxies with a (young) central stellar disk (e.g. NGC 4459, NGC 4526) or a (young) KDC (e.g. NGC 3032, NGC 4150) (Young et al. in prep.). CO also traces well the young stellar populations and ionised gas distribution and kinematics, but in KDCs not always the stellar kinematics (see Emsellem et al. 2004; Sarzi et al. 2006; Kuntschner et al. 2006).
\end{abstract}

Keywords. galaxies: elliptical and lenticular, $\mathrm{cD}$, galaxies: formation, galaxies: evolution, galaxies: kinematics and dynamics, galaxies: stellar content, galaxies: individual (NGC 2974, NGC 3032, NGC 4150, NGC 4459, NGC 4526)

\section{References}

Emsellem, E., et al. 2004, MNRAS 352, 721.

Jeong, H., Bureau, M., Yi, S.K., Krajnović, D., \& Davies, R.L. 2006, MNRAS submitted.

Kuntschner, H., et al. 2006, MNRAS 369, 497.

McDermid, R.M., et al. 2006, MNRAS submitted.

Sarzi, M., et al. 2006, MNRAS 366, 1151. 